\title{
Production matrices for geometric graphs
}

\author{
Clemens Huemer Carlos Seara Rodrigo I. Silveira \\ Departament de Matemàtiques, Universitat Politècnica de Catalunya, Spain \\ \{clemens.huemer, carlos.seara, rodrigo.silveira\}@upc.edu
}

\author{
Alexander Pilz \\ Department of Computer Science, ETH Zürich, Switzerland \\ alexander.pilz@inf.ethz.ch
}

\begin{abstract}
We present production matrices for non-crossing geometric graphs on point sets in convex position, which allow us to derive formulas for the numbers of such graphs. Several known identities for Catalan numbers, Ballot numbers, and Fibonacci numbers arise in a natural way, and also new formulas are obtained, such as a formula for the number of non-crossing geometric graphs with root vertex of given degree. The characteristic polynomials of some of these production matrices are also presented. The proofs make use of generating trees and Riordan arrays.

Keywords: geometric graph, production matrix, Catalan number, Riordan array
\end{abstract}

\section{Introduction}

This work is devoted to the prominent problem of counting geometric graphs. A geometric graph on a point set $\mathcal{S}$ is a graph with vertex set $\mathcal{S}$ whose edges are straight-line segments with endpoints in $\mathcal{S}$. It is called non-crossing if no two edges intersect except at common endpoints. Here, we only consider noncrossing geometric graphs on sets $\mathcal{S}$ of $n$ points in convex position. Already 
in 1753, Euler and Segner determined the number of triangulations (i.e., noncrossing geometric graphs with the maximal number of edges) of $\mathcal{S}$. These numbers are the well-known Catalan numbers. For many other classes of noncrossing geometric graphs (henceforth called graphs), Flajolet and Noy [6] gave formulas for their numbers. In this work, we derive such formulas by devising an $n \times n$ matrix $A_{n}$ for each of several graph classes. The numbers of these graphs on a certain number of vertices are then given by (a column of) powers of $A_{n}$. To this end, we partition the graphs on $i \leq n$ vertices according to the degree of a specified root vertex. Each part is counted in the elements of an $n$ element integer vector $\boldsymbol{v}^{i}$, and hence the sum of the elements gives the number of geometric graphs on $i$ vertices. The production matrix $A_{n}$ then gives $\boldsymbol{v}^{i+1}=$ $A_{n} \boldsymbol{v}^{i}=A_{n}^{i+1-c} \boldsymbol{v}^{c}$, when starting with a vector $\boldsymbol{v}^{c}$ for a constant number of vertices, which will usually be $(1,0, \ldots, 0)^{\top}$. To find the matrix $A_{n}$, the graphs are implicitly arranged in a tree structure (called generating tree), s.t., for each graph on $i$ vertices and with root degree $j$, the number of its descendants on $i+1$ vertices with root degree $\ell$ (for each $\ell$ ) is known. Generating trees are the basis of the ECO method [1], and have been used to obtain matrix representations for combinatorial objects $[3,9]$. For graphs on point sets in convex position, generating trees for triangulations [8] and spanning trees [7] have been obtained. We illustrate this scheme for the case of triangulations in Section 2 in some more detail, and omit the (more involved) proofs for other graph classes in this abstract. A matrix derived from succession rules is also denoted as AGT matrix [9] (matrix associated to a generating tree). We use the term production matrices (introduced in [3]). Almost all the matrices we obtain are upper Hessenberg matrices. To analyze the production matrices obtained we follow the approach of Merlini and Verri [9], using the theory of Riordan arrays. Our matrix notation is thus motivated by [9, Theorem 3.10]. A main interest of our research is

(i) to find out how the production matrices look like,

(ii) to determine the characteristic polynomial of these matrices,

(iii) and to count the number of the geometric graphs with given root vertex degree, which also implies the total number of geometric graphs.

Knowing the characteristic polynomial of a production matrix is interesting for several reasons. For instance, the Cayley-Hamilton theorem then implies a relation among the numbers of graphs with given root vertex degree. Also, it follows from the Perron-Frobenius theorem that the largest eigenvalue of the production matrix, when $n$ tends towards infinity, gives the asymptotic growth of the numbers of graphs. Precise formulas for the num- 
bers of graphs, and for the numbers of graphs with given root vertex degree are presented. We studied the following graph classes: triangulations, matchings, spanning trees, forests, spanning paths, and all geometric graphs on $n$ vertices. We mention that the production matrix for triangulations is the same as for non-crossing partitions and for perfect matchings. However, for non-crossing partitions, matchings, perfect matchings, and spanning paths, the degree of the root vertex is defined in a different way, based on visibility (we omit the definition here). For all other graph classes, the degree is the number of edges incident to the root vertex. Many of the obtained results are known; and Catalan numbers, Fibonacci numbers, Ballot numbers appear several times. We present them together with the several new results to give a more complete picture, and also since diverse results are known in a different context. Our contribution consists of some of the production matrices, the characteristic polynomials, and some of the other formulas. For example, we obtain the number of non-crossing geometric graphs with root vertex of given degree, which compares to a recent result from [4], where the distribution of the root degree of a random geometric graph is obtained.

\section{Triangulations}

The triangulations of a set of $n+2$ points in convex position are counted by Catalan numbers $C_{n}=\frac{1}{n+1}\left(\begin{array}{c}2 n \\ n\end{array}\right)$. Let us present the production matrix for triangulations, its characteristic polynomial, and outline how to derive the Catalan and Ballot numbers from this matrix. These results are not new, but are given here to illustrate the technique used also for the other graph classes, which are somewhat more involved.

Our production matrices are devised by using a surjective mapping of the graphs with $(i+1)$ vertices to the graphs with $i$ vertices. The partition of the numbers in $\boldsymbol{v}^{i}$ will depend on properties of the vertex $p_{i}$ in this mapping. We will introduce this framework with our first graph class, the triangulations.

Given a set of points $\left\{p_{1}, \ldots, p_{i+1}\right\}$ sorted counter-clockwise in convex position, Hurtado and Noy [8] defined the tree of triangulations by using the following mapping between the set $\mathcal{T}_{i}$ of triangulations on $i$ points and $\mathcal{T}_{i+1}$. For a triangulation $\tau \in \mathcal{T}_{i+1}$, one can obtain a triangulation $\tau^{\prime} \in \mathcal{T}_{i}$ by replacing every edge $p_{j} p_{i+1}$ by $p_{j} p_{i}$ (which may already exist). We call $\tau^{\prime}$ the parent of $\tau$. Observe that the number of children of $\tau^{\prime}$ depends on the vertex degree $d\left(p_{i}\right)$ of $p_{i}$, and that a child can always be obtained by adding $p_{i+1}$ and flipping edges incident to $p_{i}$.

Let $\boldsymbol{v}_{j}^{i}$ be the number of triangulations on $i \geq 3$ points s.t. $d\left(p_{i}\right)=j+1$. 
To derive $\boldsymbol{v}^{i+1}$ from $\boldsymbol{v}^{i}$, we first observe that we can add $p_{i+1}$ as a vertex of degree 2 to any triangulation of $\mathcal{T}_{i}$, and thus, $\boldsymbol{v}_{1}^{i+1}=\left|\mathcal{T}_{i}\right|$. Each triangulation in $\mathcal{T}_{i}$ has $d\left(p_{i}\right)$ children, which have a degree between 2 and $d\left(p_{i}\right)+1$ at $p_{i+1}$. Hence, $\boldsymbol{v}_{1}^{i+1}=\sum_{l \geq 1} \boldsymbol{v}_{l}^{i}$, and $\boldsymbol{v}_{j}^{i+1}=\sum_{l \geq j-1} \boldsymbol{v}_{l}^{i}$ for $j>1$. Thus, considering $\boldsymbol{v}^{i}$ as an $n$-dimensional vector with $n>i, \boldsymbol{v}^{i+1}$ can be obtained by multiplying $\boldsymbol{v}^{i}$ by a matrix $T_{n}$ as shown in Fig. 1(a).

The first column of $\left(T_{n}\right)^{i-3}$ contains the number of triangulations of a convex $i$-gon. More precisely, the entry $(j, 1)$ of $\left(T_{n}\right)^{i-3}$ is the number of such triangulations in which the root vertex has degree $j+1$, and thus entry $(1,1)$ corresponds to the Catalan number $C_{(i-3)}$.

The matrix $T_{n}$ is well known, see for instance [3]. It can also be obtained by the following succession rule from [9, Equation (2.1)]:

$$
\left\{\begin{array}{l}
\text { root }:(2) \\
\text { rule }:(k) \rightarrow(2) \cdots(k)(k+1)
\end{array}\right.
$$

This rule indicates that if root vertex $p_{i}$ of a triangulation has degree $k$, then it has one successor (child) of degree 2, one of degree 3, etc., and one of degree $k+1$. Hence, the generating tree defined by this succesion rule is equivalent to the tree of triangulations from [8].

The matrix $T_{n}$ also corresponds to a Riordan array, see [9]. Applying the results in [9] one obtains that the number of triangulations with root vertex of degree $k$ equals $\frac{k+1}{n+1}\left(\begin{array}{c}2 n-k \\ n\end{array}\right)$, known as Ballot numbers $\left(B_{n, k}\right)$.

Chow [2] determined the eigenvalues of $T_{n}$. The characteristic polynomial of $T_{n}$, also see [5], is,

$$
t_{n}(\lambda)=\sum_{j=0}^{\lceil n / 2\rceil}\left(\begin{array}{c}
n-j+1 \\
j
\end{array}\right)(-1)^{n+j} \lambda^{n-j} .
$$

Having an expression for the characteristic polynomial allows us to apply the Cayley-Hamilton theorem, which in this case tells us that $\sum_{j=0}^{\lceil n / 2\rceil}\left(\begin{array}{c}n-j+1 \\ j\end{array}\right)(-1)^{n+j}\left(T_{n}\right)^{n-j}=0$. This gives a relation among the numbers of triangulations with root vertex of degree $k$. For example, for $k=2$ (entry $(1,1)$ of $\left.T_{n}\right)$ we obtain the following relation for Catalan numbers:

$$
\sum_{j=0}^{\lceil n / 2\rceil}\left(\begin{array}{c}
n-j+1 \\
j
\end{array}\right)(-1)^{n+j} C_{n-j}=0 .
$$




\section{$3 \quad$ Other graph classes}

In this section we summarize some of the results obtained for all the graph classes studied, by presenting examples of the production matrices (Fig. 1), and expressions for the $k$ th term of the vector $\boldsymbol{v}^{n}$ (Fig. 2), for each graph class.

\begin{tabular}{|c|c|c|c|c|c|c|c|c|c|c|c|c|c|c|c|c|c|}
\hline 1 & 1 & 1 & 1 & 1 & 1 & 0 & 1 & 1 & 1 & 1 & 1 & 2 & 3 & 4 & 5 & 6 & 7 \\
\hline 1 & 1 & 1 & 1 & 1 & 1 & 1 & 0 & 1 & 1 & 1 & 1 & 1 & 2 & 3 & 4 & 5 & 6 \\
\hline 0 & 1 & 1 & 1 & 1 & 1 & 0 & 1 & 0 & 1 & 1 & 1 & 0 & 1 & 2 & 3 & 4 & 5 \\
\hline 0 & 0 & 1 & 1 & 1 & 1 & 0 & 0 & 1 & 0 & 1 & 1 & 0 & 0 & 1 & 2 & 3 & 4 \\
\hline 0 & 0 & 0 & 1 & 1 & 1 & 0 & 0 & 0 & 1 & 0 & 1 & 0 & 0 & 0 & 1 & 2 & 3 \\
\hline 0 & 0 & 0 & 0 & 1 & 1 & 0 & 0 & 0 & 0 & 1 & 0 & 0 & 0 & 0 & 0 & 1 & $2)$ \\
\hline \multicolumn{6}{|c|}{ (a) Triangulations } & \multicolumn{6}{|c|}{ (b) Matchings } & \multicolumn{6}{|c|}{ (c) Spanning trees } \\
\hline 1 & 1 & 1 & 1 & 1 & 1 & 1 & 1 & 1 & 1 & 1 & 1 & 1 & 1 & 1 & 0 & 0 & 0 \\
\hline 1 & 3 & 4 & 5 & 6 & 7 & 1 & 3 & 3 & 3 & 3 & 3 & 1 & 0 & 0 & 0 & 0 & 0 \\
\hline 0 & 1 & 3 & 4 & 5 & 6 & 0 & 2 & 4 & 4 & 4 & 4 & 0 & 1 & 0 & 0 & 0 & 0 \\
\hline 0 & 0 & 1 & 3 & 4 & 5 & 0 & 0 & 2 & 4 & 4 & 4 & 1 & 1 & 1 & 1 & 1 & 1 \\
\hline 0 & 0 & 0 & 1 & 3 & 4 & 0 & 0 & 0 & 2 & 4 & 4 & 0 & 1 & 1 & 0 & 1 & 1 \\
\hline 0 & 0 & 0 & 0 & 1 & 3 & 0 & 0 & 0 & 0 & 2 & $4)$ & 0 & 0 & 1 & 0 & 0 & $1)$ \\
\hline \multicolumn{6}{|c|}{ (d) Forests } & \multicolumn{6}{|c|}{ (e) Geometric graphs } & \multicolumn{6}{|c|}{ (f) Paths } \\
\hline
\end{tabular}

Fig. 1. Production matrices for six different graph classes, for $n=6$. Matrix (f) is for paths on at most $n / 2$ points.

As an illustrating example among our further results, we mention that the characteristic polynomial of the production matrix of spanning trees is

$$
s_{n}(\lambda)=\sum_{j=0}^{n}\left(\begin{array}{c}
2 j+2 \\
n-j
\end{array}\right) \lambda^{j}(-1)^{j}
$$

and the determinant of the production matrix of forests is a Fibonacci number.

Acknowledgments C.H., C.S., and R. I. S. were partially supported by projects MINECO MTM2015-63791-R and Gen. Cat. DGR2014SGR46. R. I. S. was also supported by MINECO through the Ramón y Cajal program.

\section{References}

[1] Barcucci, E., A. D. Lungo, E. Pergola and R. Pinzani, ECO: a methodology for the enumeration of combinatorial objects, J. Differ. Equations Appl. 5 (1999), pp. $435-490$.

[2] Chow, T. S., A class of Hessenberg matrices with known eigenvalues and inverses, SIAM Rev. 11 (1969), pp. 391-395, mR 405627. 


\begin{tabular}{|l|l|}
\hline Triangulations & $B_{n, k}=\frac{k+1}{n+1}\left(\begin{array}{c}2 n-k \\
n\end{array}\right) \quad\left(B_{n, k}\right.$ are Ballot numbers $)$ \\
\hline Matchings & $\sum_{j=k-1}^{n}\left(\begin{array}{c}n \\
j\end{array}\right)(-1)^{n+j} B(j, k-1)$ \\
\hline Spanning trees & $\frac{k}{n-1}\left(\begin{array}{c}3 n-k-4 \\
n-k-1\end{array}\right)$ \\
\hline Forests & $\frac{1}{n-1} \sum_{j=0}^{n-1}\left(\begin{array}{c}n-1 \\
j\end{array}\right)\left(k\left(\begin{array}{c}n+2 j-k-2 \\
j-k\end{array}\right)+(k+1)\left(\begin{array}{c}n+2 j-k-3 \\
j-k-1\end{array}\right)-(k+2)\left(\begin{array}{c}n+2 j-k-4 \\
j-k-2\end{array}\right)\right)$ \\
\hline Geometric graphs & $\left.\frac{2^{n-2}}{n-1} \sum_{j=0}^{n-1}\left(\begin{array}{c}n-1 \\
j\end{array}\right)\left(\begin{array}{c}n+j-k-2 \\
j-k\end{array}\right)-(k+2)\left(\begin{array}{c}n+j-k-4 \\
j-k-2\end{array}\right)\right)$ \\
\hline Paths & {$\left[2^{n-k-2}+(n-k) 2^{(n-k-3)}\right]$ for $k=1, \ldots, n-1$} \\
\hline
\end{tabular}

Fig. 2. Formulas for the number of graphs for each class with $n$ vertices in which the root vertex has degree $k$. (The degree is defined in terms of visibility for matchings and paths). Some are only valid for $n>1$ and $k>0$. For paths, the formula corresponds to the sum of two entries of the vector.

[3] Deutsch, E., L. Ferrari and S. Rinaldi, Production matrices, Adv. in Appl. Math. 34 (2005), pp. 101-122.

[4] Drmota, M., A. de Mier and M. Noy, Extremal statistics on non-crossing configurations, Discrete Mathematics 327 (2014), pp. 103-117.

[5] Fairweather, G., On the eigenvalues and eigenvectors of a class of Hessenberg matrices, SIAM Rev. 13 (1971), pp. 220-221.

[6] Flajolet, P. and M. Noy, Analytic combinatorics of non-crossing configurations, Discrete Mathematics 204 (1999), pp. 203-229.

[7] Hernando, M. C., F. Hurtado, A. Márquez, M. Mora and M. Noy, Geometric tree graphs of points in convex position, Discrete Applied Mathematics 93 (1999), pp. 51-66.

[8] Hurtado, F. and M. Noy, Graph of triangulations of a convex polygon and tree of triangulations, Comput. Geom. 13 (1999), pp. 179-188.

[9] Merlini, D. and M. C. Verri, Generating trees and proper Riordan arrays, Discrete Mathematics 218 (2000), pp. 167-183. 